\title{
Development of ABS $\mu$ controller under the influence of
}

\author{
overload perturbation \\ Chaochun Yuan ${ }^{1 a}$, Yiqun Liu ${ }^{2 b}$, Fei Wu²c \\ 1.Automotive Engineering Research Institute ,JiangSu University, \\ Zhengjiang ,Jiangsu 212013, China \\ 2.School of Automobile and Traffic Engineering, Jiangsu University, Zhenjiang, \\ Jiangsu 212013, China \\ a.277062332@qq.com b.liuyiqun1991512@163.com c.23432432@qq.com
}

Key words: $\mu$ theory; overload perturbation;Anti-lock braking;Chery vehicle

\begin{abstract}
In order to improve the control effect and robust performance of ABS under overload perturbation. A fourth dynamics perturbation model and brake perturbation model are established. Then $\mu$ theory is used to design the ABS $\mu$ controller through $\mathrm{D}-\mathrm{K}$ iteration.A Chery vehicle is taken as the research object,simulation and ABS hardware in loop experiments are designed to test the robustness of $\mu$ controller compared with $H_{\infty}$ controller. The results show that compared with $H_{\infty}$ controller, designed $\mu$ controller can reduce the impact of overload perturbation and external noise effectively.
\end{abstract}

\section{Introduction}

Anti-lock braking system(ABS) has several advantages compared with tradition brake system. When under emergency braking situation, it can control the slip rate around the desired value to make full use of the peak longitudinal adhesion coefficient,especially on slippery roads. While, the development of control strategy plays an important role in $\mathrm{ABS}[1,2,3,4]$.It is the core of the anti-lock braking system.

In recent years a lot of theoretical research has been done on anti-lock braking system control algorithms by many scholars.Beak Seunghwan from Korea and Kazemi from Iran Vehicle Dynamics Department designed the sliding model ABS controllers[5,6]. These controllers improve the response time and the safety on the slippery road. Chen $\mathrm{C}-\mathrm{K}$ from China and Mirzaei A from Isfahan University of Technology adopted a fuzzy ABS controller based on fuzzy theory[7,8,9], which increased the flexibility of the braking system. However,all these control algorithms do not consider the parameter variations caused by overload as well as the external sensor noise disturbance in actual use. Such perturbations may lead to the overshoot and long setting time or even the unstable of the system. While, in $\mu$ theory[11], input,output,transfer function and perturbation are reconstructed to isolate all the uncertainties and perturbation, so it can better solve the deterioration of the control performance and system instability caused by parameter perturbation in closed-loop system.

In this paper, we study a Chery vehicle with hydraulic transmission ABS system and consider the parameter perturbation effect.Then, Linear fractional 
transformations(LFT) is applied to analyze the perturbation. $\mu$ controller for ABS is designed based on $\mu$ theory, and compared with $H_{\infty}$ controller through simulation and hardware in loop experiments.

\section{Perturbation characteristic analysis and model of ABS system}

System perturbation Characteristics.In actual use,the different car load result in the variation of quality. It is obvious that this parameter perturbation can affect the performance of the vehicle braking system directly. In order to solve the impact of parameter perturbation, linear fractional transformation(LFT) is used to deal with changes. The LFT equations are defined as follows:

$\frac{1}{M}=\frac{1}{\bar{M}\left(1+d_{M} \delta_{M}\right)}=F_{l}\left\{\left|\begin{array}{cc}\frac{1}{\bar{M}} & -\frac{d_{M}}{M} \\ 1 & -d_{M}\end{array}\right|, \delta_{M}\right\}$

Where $\bar{M}$ is the nominal value of the corresponding parameter, $d_{M}$ represents the degree of perturbation parameters, with the range from 0 to $1, \delta_{M}$ express the unit value perturbation, with the range from -1 to 1 .

Dynamics perturbation vehicle model. The development of a vehicle dynamics is an important step towards the development of ABS strategy. One quarter, two dimension dynamics vehicle equations is built to design and research the basic strategies. It is assumed that :

(1)Automotive quality is distributed in each wheel evenly.

(2)When travel along a straight line, there is no question of the tire lateral force.

According to D'Alembert's Principle, and consider variations in vehicle quality, dynamics perturbation equations are established as follows:

$$
\begin{aligned}
& \bar{M}\left(1+d_{M} \delta_{M}\right) \dot{v}=-F-G f \cos \alpha-\frac{C_{D} A}{21.15} u^{2}-G \sin \alpha \\
& I \dot{\omega}=F * R-T_{b} \\
& F=\mu N
\end{aligned}
$$

Where $M$ is $1 / 4$ vehicle normal mass, $d_{M}$ and $\delta_{M}$ are the degree of perturbation in mass and the unit value perturbation respectively, $v$ and $\omega$ represent velocity and angular velocity separately, $T_{b}$ is brake friction torque, $R$ is radius of tire, $I$ stands for moment of inertia of tire, $\mu$ and $\alpha$ denote the longitudinal adhesion coefficient and road slope angle respectively, air resistance coefficient and normal force are marked by $C_{D}$ and $N, A$ is windshield area of the vehicle.

Tire model.According to the experiments, when the road and type of the tire have been given, adhesion coefficient is mainly determined by slip rate and speed. But speed has a small influence on the adhesion coefficient, the absolute difference of peak adhesion coefficient between $10 \mathrm{~km} / \mathrm{h}$ and60 $\mathrm{km} / \mathrm{h}$ is only 0.05 .

Burckhardt proposed a tire model[10], which reflects the characteristics of the road adhesion coefficient truly.In this model longitudinal adhesion coefficient is expressed as a function of slip rate.Its definition is as follows:

$$
\mu(s)=B\left(1-e^{-C s}\right)-D s
$$

Where B,C,D are parameters associated with the road.

The corresponding parameter of dry concrete road are showed as follows:

$B=0.956 ; C=20.822 ; D=0.21$

Hydraulic transmission model.A mode of hydraulic transmission system is showed in Fig. 2 to analyze the factors which affect the characteristics of pressure increase, 
pressure hold and pressure decrease of wheel cylinder as well as the lag characteristics of pressure switching state.

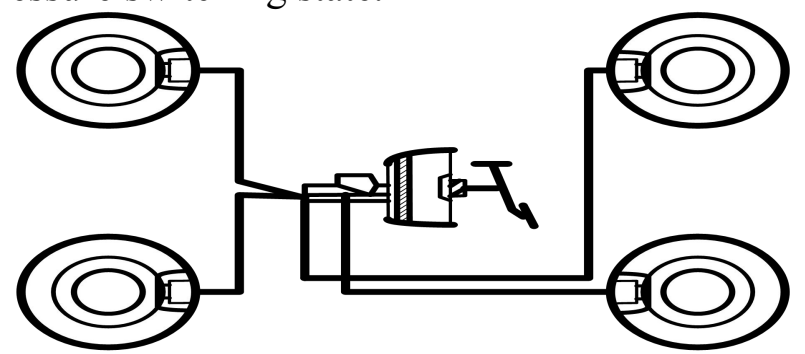

Fig.2hydraulic transmission system

When brake pressure increases, continuity equation of flow pressure variation is defined as:

$Q_{w}=\frac{V}{K} \frac{d p_{w}}{d t}$

Where $K$ is the elastic modulus of the volume of oil and $V$ is the total volume of brake cylinder and pipes.

According to the consequence of pressure increase, the change rate of pressure decrease can also be depicted as follows:

$\frac{d p_{w}}{d t}=\frac{C_{B} A_{B} K}{V} p_{w}^{\varphi}$

Where $C_{B}, A_{B}$ and $\varphi$ are the related coefficient of normally closed valve under pressure decrease stage.

Except the throttling effect generated by pressure difference of the two sides of the valve, the hydraulic transmission system still exists lag characteristics and the time can be calculated as follows:

$\tau=t_{\text {on/off }}+t_{\text {deliver }}$

Where $t_{\text {on/off }}$ is the opening or closing time of the electromagnetic valve and $t_{\text {deliver }}$ is the travel time of pressure.

In summary, the hydraulic transmission dynamic model can be depicted as:

$\frac{d p_{w}}{d t}=\frac{C_{T} A_{T} K}{V}\left(p_{m}-p_{w}\right)^{k} u_{1}\left(t-\tau_{w p}\right)-\frac{C_{B} A_{B} K}{V} p_{w}{ }^{\varphi} u_{2}\left(t-\tau_{w p}\right)$

Where $\tau_{w p}$ is the system lag time, $u_{1}$ and $u_{2}$ are the control signal of the electromagnetic valve.

Brake model. When the brake cylinder pressure model is made, mathematical expressions are needed to describe the functional relationship between the wheel cylinder pressure and the hydraulic brake torque. The force in the brake disc is showed in Fig.3.The relationship between the pressure applied by brake cylinder piston to friction plate and wheel cylinder pressure are defined:

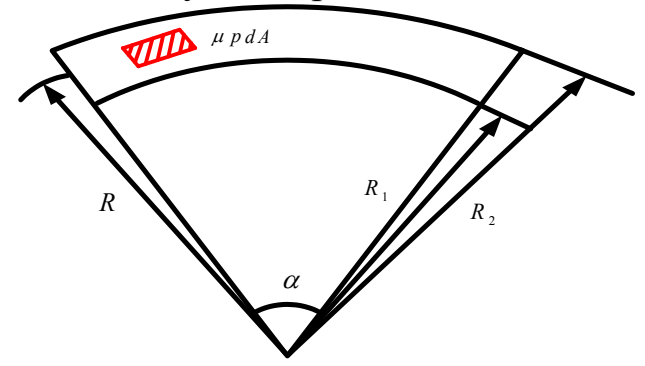

Fig.3: Force analysis of the brake disc

$N_{p}=p_{w} g A_{m c}$ 
Where $p_{w}$ represents the wheel cylinder pressure, $N_{p}$ is the pressure forced by piston to the friction plate and cross-sectional area of friction plate is marked by $A_{m c}$.

Take a tiny cell area in the brake disc, the friction it has is $k_{p} p d A$, where $k_{p}$ represents the friction coefficient between friction plate and break disc.

Due to two outer sides of brake disc have force, so the hydraulic brake torque it has can be expressed as:

$T_{b h}=2 \int_{R_{1}}^{R_{2}} 1 \int_{0}^{\phi} R k_{p} p d A=\frac{2 k_{p} p \phi}{3}\left(R_{2}^{3}-R_{1}^{3}\right)$

\section{System $\mu$ controller design}

The block scheme of ABS is depicted in Fig.4 according to the the $\mu$ synthesis theory. In this feedback system, mass is considered as perturbation factors, $s^{*}$ is the desired slip rate, and $\Delta_{M}$ denotes the mass perturbation.It is stable and satisfy $\left\|\Delta_{M}\right\|<1$. The input and output of them are marked by $z_{1}, w_{1}$.

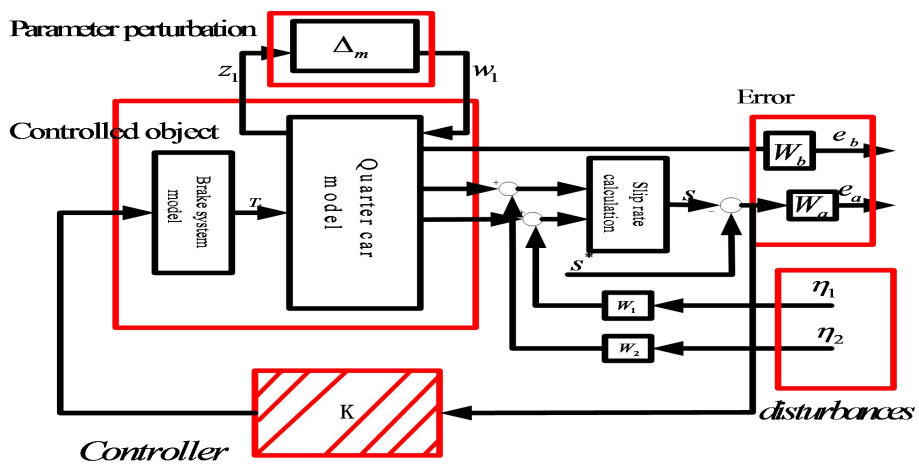

Fig.4: Structure diagram of brake control system

Controller design rule.As mentioned before,evaluation errors are used to evaluate the robust performance of the whole control system. In this system,slip rate error and deceleration are designed as the performance errors and slip rate error should remain reasonably small in the face of the exogenous signals and the parameter perturbation, so the following detail rules are designed:

(1)Reduce $\left\|W_{a}\left(s-s^{*}\right)\right\|_{2}$ to get optimal slip ratio, so that the system can have better braking performance.

Where $W_{a}$ is the weighting function, and it has larger amplitude in low frequency and appropriate gain in high frequency to control overshoot. Its structure are defined as $W_{a}=\frac{a s+b}{c s+d}$, and $a, b, c, d$ are decided through control performance.

(2)To ensure comfort, the maximum braking deceleration should not be too large, so $W_{b}$ are designed as $1 / 8$, to make sure the maximum braking deceleration is less than $7 \mathrm{~m} / \mathrm{s}^{2}$.

Weighting function selection. The external disturbances of the ABS include vehicle speed and angular velocity sensor noise. $\eta_{1}$ and $\eta_{2}$ are input of these sensor noise respectively. $W_{1}$ and $W_{2}$ are the corresponding weighting function. $W_{1}$ is designed as: $\frac{0.17 s+0.03}{s+1}$, which indicates the measuring noise of speed in low frequency is $0.03 \mathrm{~m} / \mathrm{s}$, while in high frequency it is $0.17 \mathrm{~m} / \mathrm{s} . W_{2}$ is designed as: $\frac{0.34 \mathrm{~s}+0.012}{0.5 \mathrm{~s}+0.1}$, which shows the measuring noise of angular velocity in low frequency is $0.12 \mathrm{rad} / \mathrm{s}$, while in 
high frequency is $0.68 \mathrm{rad} / \mathrm{s}$.After calculation in Matlab $\mu$ tool box, $W_{a}$ is selected as $\frac{2 s+0.7}{s+0.04}$ and it can ensure the steady-state error less than $6 \%$.

$\mu$ synthesis control algorithm. $\mu$ synthesis control algorithm is the minimize loop system in all stable controllers.It can be depicted as follows:

$\min \max \mu_{\Delta}\left\lfloor F_{l}\left(P, K_{\mu}\right)(j \omega)\right\rfloor$

Where $K_{\mu}$ is stable, $D(s)$ is introduced to represent the scale changes matrix, $D(s)$ is a stable and reversible minimum phase matrix. $D_{\omega}$ is the element of scale changes matrix $D(s)$ and $D_{\omega}$ is independent in each $\omega$.Formula(16) can also be written as:

$\min \min \left\|\left[D_{\omega} F_{l}\left(P, K_{\mu}\right)(j \omega) D_{\omega}\right]^{-1}\right\|_{\infty}$

To the system perturbation $\Delta_{M}$, a stable controller $K_{\mu}(s)$ can be solved to keep the system stable under parameters perturbation. D-K iteration method is used in solving $\mu$ controller $K_{\mu}(s)$. In D-K iteration method, $\mu$ robust controller is designed to seek stable control matrix and scale changes matrix $D(s)$. The optimized target for the system is defined as follows:

$\inf _{D, K_{\mu}} \sup \sigma\left(D F_{l}\left(P, K_{\mu}\right) D^{-1}\right)=\inf \left\|D F_{l}\left(P, K_{\mu}\right) D^{-1}\right\|_{\infty}$

Hold $D(s)$ unchanged, so equation(15) is a $H_{\infty}$ optimal problem, it can be solved by Riccta equation. Then hold $K_{\mu}(s)$ unchanged,optimize $D(s)$ based on the known stable controller $K_{\mu}(s)$.

\section{Simulation analysis}

A Chery vehicle with hydraulic disc brake is taken as the study object. Disconnect the uncertainty module in Fig.4 and select the weighting functions, a open loop system is got. Then $\mu$ control box is used to solve the $\mu$ controller for the open loop structure of the ABS system. The main parameters of the model for solving controller simulation analysis and experiments are showed on the table.1.

Table 1: Main parameters of system

\begin{tabular}{|c|c|c|}
\hline Description & Symbol & Value \\
\hline 1/4 vehicle mass & $M$ & $400 \mathrm{~kg}$ \\
\hline Moment of inertia of the wheel & $I$ & $2.5 \mathrm{kgm}^{2}$ \\
\hline Wheel radius & $R$ & $0.3 \mathrm{~m}$ \\
\hline Gravitational constant & $G$ & $9.8 \mathrm{~m} / \mathrm{s}^{2}$ \\
\hline friction coefficient & $k_{p}$ & $178 \mathrm{Nm} / \mathrm{MPa}$ \\
\hline road slope angle & $\alpha$ & $2^{\circ}$ \\
\hline Air resistance coefficient & $C_{D}$ & $0.379 \mathrm{~kg} / \mathrm{m}^{3}$ \\
\hline windshield area & $A$ & $1.8 \mathrm{~m}^{2}$ \\
\hline
\end{tabular}

Time simulation analysis.Based on the $\mu$ synthesis algorithm, after 2 iterations a 8 order controller is got. In order to assess the robustness of the $\mu$ controller, a $H_{\infty}$ controller is designed based on the $H_{\infty}$ theory for the structure mentioned above without changing the input and output.The reference on $H_{\infty}$ theory can be seen on[12]. 
According to GB 21670-2008 Passenger car brake system technical requirements and test methods the Simgui tool in Matlab is used to simulate the emergency braking with the initial speed at $90 \mathrm{~km} / \mathrm{h}$ in high adhesion road. Road adhesion coefficient is assumed as 0.85 and ideal slip rate is 0.2. Senor noise is supposed as random measurement noise with 0.01 covariance.

Figure. 5 and 6 describe the time response of the vehicle speed, tire angular velocity and the change of brake time of the two controllers under $30 \%$ increase in mass respectively. It can be seen from fig. 6 that the brake effect of $\mu$ controller is much better than $H_{\infty}$ controller, the brake time of $\mu$ controller is only $3.8 \mathrm{~s}$, almost the same as that under normal case. However, the brake time of $H_{\infty}$ controller is $4.4 \mathrm{~s}, 0.6 \mathrm{~s}$ longer than $\mu$ controller. Fig. 5 also indicates that the range of tire angular velocity of $\mu$ controller is smaller than $H_{\infty}$ controller.

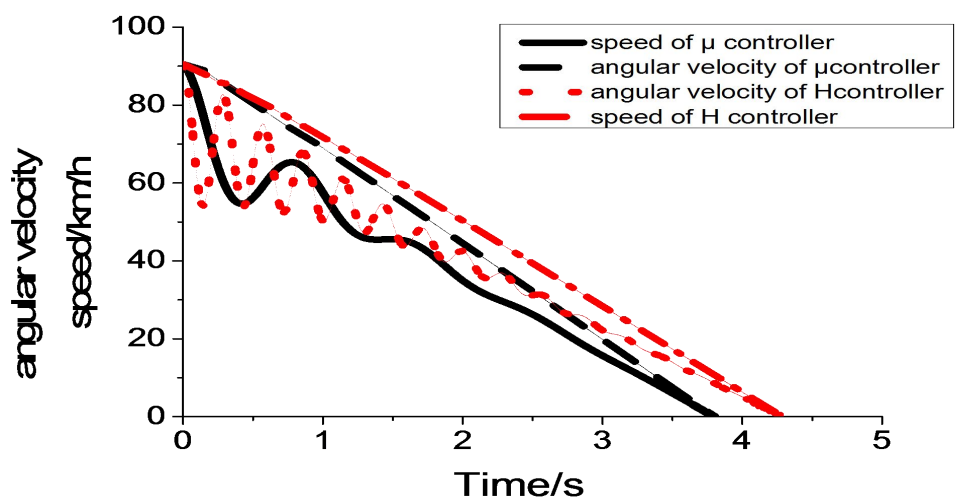

Fig.5:Simulation curve of ABS under $30 \%$ increase in mass

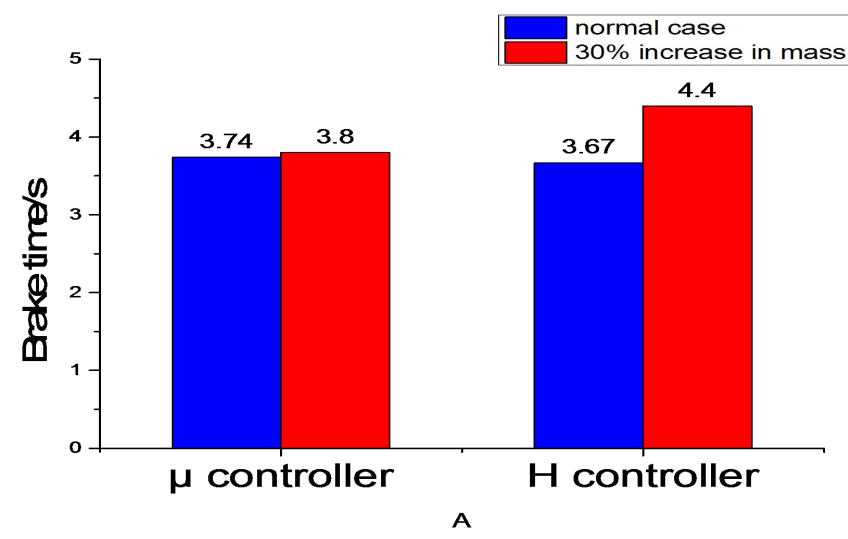

Fig.6: Brake time of two controllers

\section{Experimental Verification}

To further verify the accuracy of the model and the actual reliability of the controller. Matlab and LabVIEW software are applied to build the hardware in loop(HIL) ABS test platform based on the Chery vehicle with hydraulic disc brake. Simulation software is written by LabVIEW to build the virtual controller so that the ABS system can be monitored and controlled. Data acquisition card is used to connect the controller and the brake system bus which can exchange the brake pressure, brake pedal displacement, angular velocity and hydraulic valves signals. After receiving data, the brake system transfer real time vehicle parameters to controller then the 
controller sends control signal to achieve the brake torque control. The nominal mass of the platform inertial wheel is calculated according to the $1 / 4$ mass of that vehicle.Adjust the mass of wheel is used to change the vehicle quality .The pictorial diagram of ABS HIL bench is showed in figrue7.

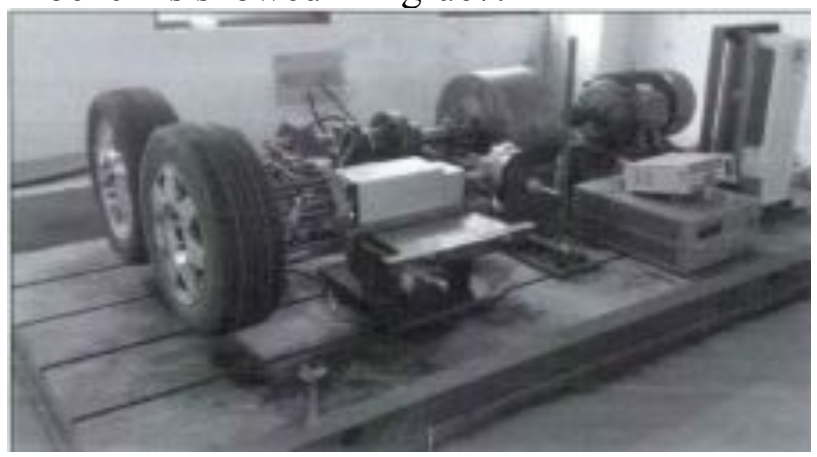

Fig.7: ABS HIL bench

The initial speed is $90 \mathrm{~km} / \mathrm{h}$ and the road adhesion coefficient is 0.85 , the brake system is controlled by the designed $\mu$ controller and $H_{\infty}$ controller to have emergency brake under 30\% increase in mass Fig. 8 shows the time response of speed and angular velocity.It is clear that under overload experimental conditions, $\mu$ controller obtains better control effect than $H_{\infty}$ controller. When there is $30 \%$ increase in mass, it takes $\mu$ controller 3.83 s to stop. While, it takes $H_{\infty}$ controller $4.49 \mathrm{~s}$, which is $0.66 \mathrm{~s}$ longer than $\mu$ controller.

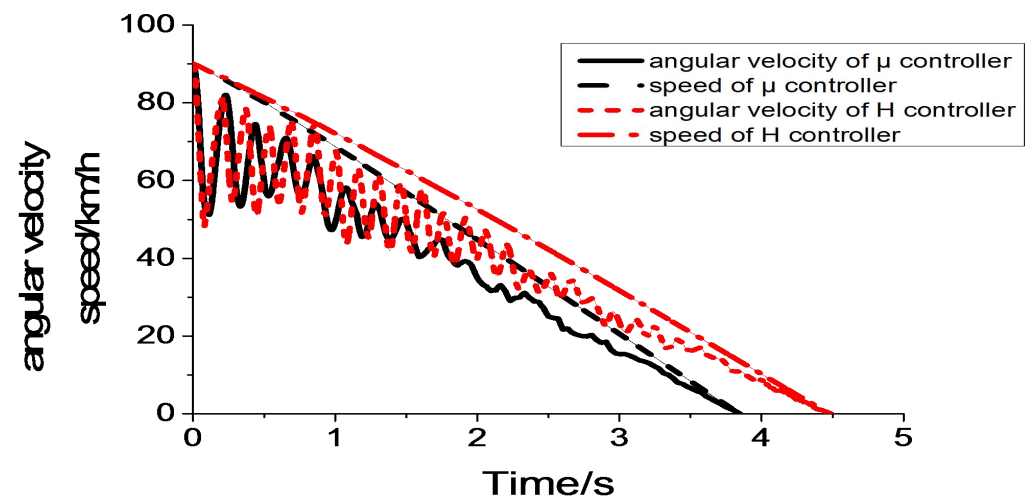

Fig.8: Experimental curve of ABS under 30\% increase in mass

\section{Summary}

This paper proposes a $1 / 4$ vehicle dynamics model and brake model with perturbation factors.By using linear fractional transformation and $\mu$ synthesis control, a $\mu$ controller is designed for the ABS system. A combination of both simulation and hardware in loop experiments are applied to test the effect of the $\mu$ controller compared with $H_{\infty}$ controller. Following specific conclusions can be drawn from the current work:

(1)The $\mu$ controller has better control effect than $H_{\infty}$ controller under the overload perturbation. When there is a perturbation, the performance deteriorates greatly under the control of $H_{\infty}$ controller.

(2)On the control of the slip rate, when there is a parameter perturbation, $\mu$ controller 
can achieve the desired slip rate with smaller steady-state error. While, $H_{\infty}$ controller can also reach the desired slip rate but the overall deviation is larger and the overshoot is greater.

Acknowledgement:

This work is supported by National Natural Science Foundation of China No.51305167 and "Six Talents Peaks" in Jiangsu Province No.2012-ZBZZ-029.The authors would like to thank the reviews for their valuable comments and the editors for improving the quality of the paper.The Corresponding author is Yiqun Liu and his E-mail address is liuyiqun1991512@163.com.

\section{Reference:}

[1]Chen,Chih-Keng,Hwang,Chi-Pan.Implementation of a PC-based ABS with CAN-bus interface on experiment platform.International Journal of Vehicle Design.2005.vol.37.pp.343-357.

[2]Liu Lizhuo,Xie Lili.An observer based adaptive control method of braking system.Journal of Computation System.2014.vol.10.pp.9237-9244.

[3]Yu Hai, Extremum-seeking control strategy for ABS with time delay.Proceedings of the American Control Conference.2002.vol.5.pp.3753-3758.

[4]Cabasino,Maria Paola.Diagnosability analysis of an ABS system modeled using Petri nets.IFAC proceedings Volumes.2012.vol.8.pp.842-847.

[5]Zheng Xihua,Liu Lizhuo.An adaptive controller for improving efficiency of aircraft braking process. Journal of Computation System. 2015. Vol.11. pp. 3883-3890.

[6]Dincmen Erkin. Extremum-seeking control of ABS braking in road vehicles with later force improvement.IEEE.Transaction on control system technology.2013.vol.22.pp.230-237.

[7]Mirzaei A, Moallem M, Dehkordi B M, Design of an Optimal Fuzzy Controller for Antilock Braking Systems. IEEE Transactions on Vehicular Technology, Vol. 55.pp.1725-1730

[8]Chen,C-K,Wang,Y-C, Fuzzy control for the anti-lock braking system.Proceedings of the Asian Fuzzy Systems Symposium.2006.vol.23.pp.67-72.

[9]Zhang Pen.Multi-field modeling and simulation of ABS based on fuzzy control.Journal of Beijin Institute of China.2014.vol.23.pp.66-71.

[10]Zheng Tai-Xiong,Ma Fu-Lei.Automotive ABS control strategy based on logic threshold.Journal of Traffic and Transportation Engineering.2010.vol.10.

pp.69-74.

[11]Packard A, D oye 1 J. The comp lex structured singu larva lue. Automatica, 1993.vol.29.pp. 71- 109.

[12]Limebeer D J N Kasenallg E. $H_{\infty}$ optimal control of a synchronous turbo generator.Proceedings IEEE CDC,Athens, 1986:62-65. 